\section{An Excel macro for transformed and weighted averaging}

\author{
STANLEY A. KLEIN \\ University of California, Berkeley, California
}

\begin{abstract}
An Excel macro is presented for averaging spreadsheet data. The macro has several special features: (1) The data are weighted by the inverse variance of each datum to decrease the contributionof noisy outliers. (2) There is a provision for a power or a log trano form of the data before averaging. The rationale for transforming the data before averaging is discussed. (3) The output includes the average value, its standard error, and the reduced chi-square that measures the goodness of fit. (4) The standard error is corrected by a heterogeneity factor based on the reduced chi-square. The averaging of data is rarely done properly, and the intent of this article is to clarify the issues and provide a tool that allows researchers to improve their averaging techniques.
\end{abstract}

Most researchers average data. Averaging seems to be a straightforward process, and few give it much thought. Yet seldom is it done well. This article represents an attempt to show that averaging can raise interesting and subtle problems. As will be discussed, there are many circumstances in which a nonlinear transform should be applied to data before averaging. There are also circumstances in which a heterogeneity factor should be applied to the standard error ( $S E$ ) of the averaged data. An Excel macro for the Macintosh or IBM is presented that is sufficiently flexible to handle these different circumstances.

\section{Illustrative Data}

To make the discussion of the various features of the Excel macro concrete, I have invented a data set that is tabulated in Figure 1 and plotted in Figure 2. The measured data (column $C$ of Figure 1) can be thought of as being one observer's detection threshold for seeing a grating with spatial frequencies of $.5,1,2$, and 4 cycles per degree. In the macro and in this article, the spatial frequency is called the $x_{-}$value (column $A$ in Figure 1 ). The dependent and independent variables need not be threshold as a function of spatial frequency. The data in Figure 1 could, for example, be the relative temperature of a room as a function of the time after an air conditioner is turned on.

An averaging routine is desirable in order to clean up Figure 2 by plotting a single datum per spatial frequency. The data are presented in Excel spreadsheet format in Fig-

\footnotetext{
This research was supported by Grant R01 EY04776 from the National Eye Institute and Grant AFOSR 89-0238 from the Air Force Office of Sponsored Research. Donna Ellinger, Thom Carney, and Scott Stevenson provided helpful comments. Correspondence should be addressed to S. A. Klein, School of Optometry, University of California, Berkeley, CA 94720.
}

ure 1 , to show how data should be entered for calculating averages. The purpose of the number in cell $\mathrm{A} 2$ will be discussed later. Column A shows that the threshold for the $x_{\text {_n }}$ value of .5 was measured four times; thresholds for the $x$ _. values of 1,2 , and 4 were measured three times apiece. Column $B$ is some other variable, such as the color of the display, that may or may not affect threshold. It is not used by the macro; it is included in Figure 1 to make the point that other columns of the spreadsheet can be filled without affecting the algorithm. Column C is the detection threshold in percent contrast. Column D is the $S E$ of the threshold estimate. The $S E$ can be calculated in several ways. For example, a large number of Monte Carlo calculations based on binomial statistics could be used to simulate the psychophysical procedure used to estimate threshold. Another way to obtain an estimate of the $S E$ is to have each measurement (each run) shown in Figures 1 and 2 consist of several submeasurements (trials) (this would be the method used for estimating the $S E$ when using the method of adjustment). Thus, the threshold of 2.1 in cell $\mathrm{C} 2$ and the $S E$ of .2 in cell D2 might have been obtained by repeating 10 measurements. The numbers in columns E-H of Figure 1 are the averaged results from the output of the macro, as will be discussed later.

Four examples will now be discussed, to illustrate several issues that one must consider when averaging data.

Example 1: Noisy outliers. The data with the $x_{\text {_ }}$ value of .5 illustrate the need for a weighted average. For the first three runs, the $S E$ s are .2 , about $10 \%$ of the threshold. The fourth run is an outlier with a large $S E$. The large $S E$ could have occurred for a variety of reasons. Maybe the observer fell asleep in the middle of a run, resulting in fewer trials for that run. Maybe the stimulus strength was too strong or too weak, with trials positioned at a shallow part of the psychometric function, so that the resulting threshold had a large $S E$. In some cases, the aberrant run could be thrown out on the basis of obvious circumstances (falling asleep). In other cases, however, the cause of the erroneous datum may not be obvious, and it would be desirable for the Excel macro to take the $S E$ into account to decrease the contribution of noisy outliers (outliers tend to have larger $S E$ s).

There are situations in which the averaging can be done simply by lumping together all the trials from the different runs before calculating thresholds. This procedure properly places heavier weight on the runs with more trials. In our signal detection methodology, however, it is not possible to simply concatenate all of the raw data, since the test contrast and also the subject's criterion (false alarm rate) may vary between runs.

Example 2: Heterogeneity factor. The data with the $x_{\text {_nalue }} 1$ illustrate the presence of heterogeneity in the data. In this case, $S E=.1$ for all three data points, but the variability from run to run $(1.2, .8,1.0)$ has a 
Data for Average.macro

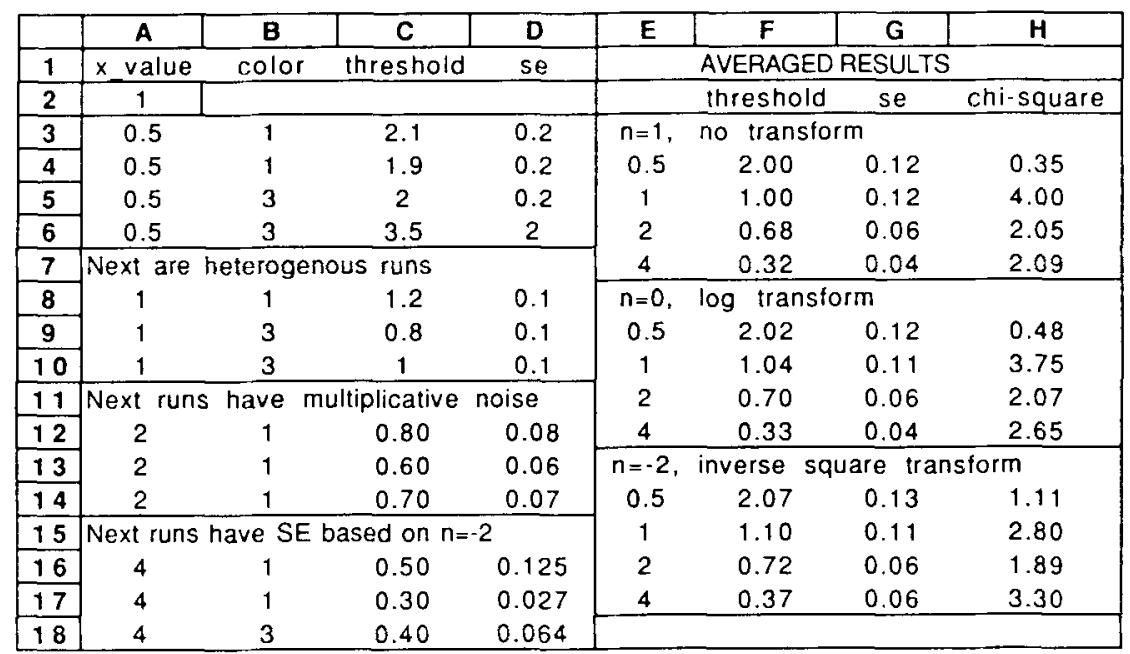

Figure 1. An Excel worksheet with sample input data in columns A-D and averaged results in columns $\mathbf{E}-\mathrm{H}$. The reason for averaging is to plot the data with only one datum for each

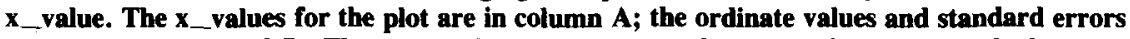
are in columns $C$ and $D$. The data to be averaged must be grouped together with the same $x_{\text {_ }}$ value. The standard errors have been chosen to illustrate several points discussed in the text. The macro was run three times, with transformation exponents of 1,0 , and -2 , to obtain the averaged thresholds and standard errors (ses) shown in columns $F$ and $G$. The reduced chisquare in column $H$ provides a measure of the goodness of fit.

greater standard deviation than would be expected from the $S E$ values, possibly due to a day-to-day fluctuation in sensitivity. It would be desirable for the Excel macro to include a heterogeneity factor in its calculation of the $S E$ of the averaged data.

Example 2 can be understood in terms of a model in which the threshold is given by

$$
t_{i}=t_{0}+n_{\mathrm{w}}+n_{\mathrm{b}}
$$

where $t_{0}$ is the noise-free threshold, $n_{w}$ is the noise within a run ( $S E=.1$ in our example) due to binomial variability and to fluctuating sensitivity within the run, and $n_{\mathrm{b}}$ is the heterogeneous noise (changing sensitivity) between runs. In Example 2, the deviations between runs of \pm .2 are significantly larger than the within-run $S E$ of .1 .

Example 3: Additive versus multiplicative noise. The data with the $x$ _value of 2 have an $S E$ equal to $10 \%$ of the threshold. Example 3 can be understood in terms of a model in which the threshold is given by

$$
t_{i}=\left(t_{0}+n_{\mathrm{w}}\right)\left(1+n_{\mathrm{b}}\right) \text {. }
$$

Equation 2 is different from Equation 1 only in that the between-run noise is multiplicative rather than additive. With multiplicative noise, the $S E$ tends to be proportional to the magnitude of the datum.

It is instructive to take the natural logarithm of the data in Example 3. The three data values become

$$
\begin{aligned}
& y_{1}=-.223 \pm .1, \\
& y_{2}=-.511 \pm .1,
\end{aligned}
$$

and

$$
y_{3}=-.357 \pm .1 \text {, }
$$

where $y=\ln (t)$ and $S E_{y}=S E_{t} / t$. The $S E$ s of the transformed data $\left(S E_{y}\right)$ equal the percent error (or fractional error) of the original data. Thus, multiplicative noise in the original data corresponds to additive noise (independent of threshold as in Equation 1) for the log of the data. The averaging of the logarithm of the data is commonly called geometric averaging, whereas the averaging of the raw data directly is commonly called arithmetic averaging.

Example 4: Power transformation. In certain circumstances, one might want to transform the data according to a power function rather than a logarithmic function. The data with the $x_{\text {___ value of }} 4$ have an $S E$ equal to the threshold raised to the third power. In this section, I will show that for this type of data it is sensible to raise each datum to the -2 power before averaging. As an example of how a power function relationship might occur, consider the signal detection connection between $d^{\prime}$ and threshold. Klein and Stromeyer (1980) showed that near threshold the transducer function is approximately a power function with an exponent of $n=2$ :

$$
d^{\prime} \approx(\operatorname{stim} / t)^{n},
$$

where stim is the stimulus strength, $t$ is the threshold (the third column of Figure 1), and $n$ is the transducer exponent. Let us suppose that the stimulus strength is fixed and $d^{\prime}$ is experimentally measured. The threshold can then be calculated from Equation 4: 


$$
t=\operatorname{stim}\left(d^{\prime}\right)^{-1 / n}
$$

The $S E$ of the threshold estimate is obtained by taking differentials of both sides of Equation 4 (Bevington, 1969):

$$
S E_{t} \approx \text { const } t^{n+1} S E_{d^{\prime}} \text {. }
$$

The important question for our present concern is whether it is better to average the threshold measurements weighted by $1 / S E_{t}^{2}$ or to average the $d^{\prime}$ measurements weighted by $1 / S E_{d^{\prime}}^{2}$. Let us assume that $d^{\prime}$ values are normally distributed and, furthermore, that $S E_{d^{\prime}}$ is constant, independent of $d^{\prime}$ (see Appendix A of Klein, Stromeyer, \& Ganz, 1974, for a justification of these assumptions). The distribution of threshold values, on the other hand, will be strongly skewed ( $d^{\prime}$ values near zero would cause extreme threshold outliers). Given the wellbehaved $d^{\prime}$ distribution, it would be advisable to average the $d^{\prime}$ values rather than the threshold values by transforming the data to ${ }^{\prime} d^{\prime}$ units by using Equation 4 . Because of the knowledge that the transducer function is quadratic $(n \approx 2)$, a power function transformation is desired for averaging. The Excel macro is able to handle this type of transformation.

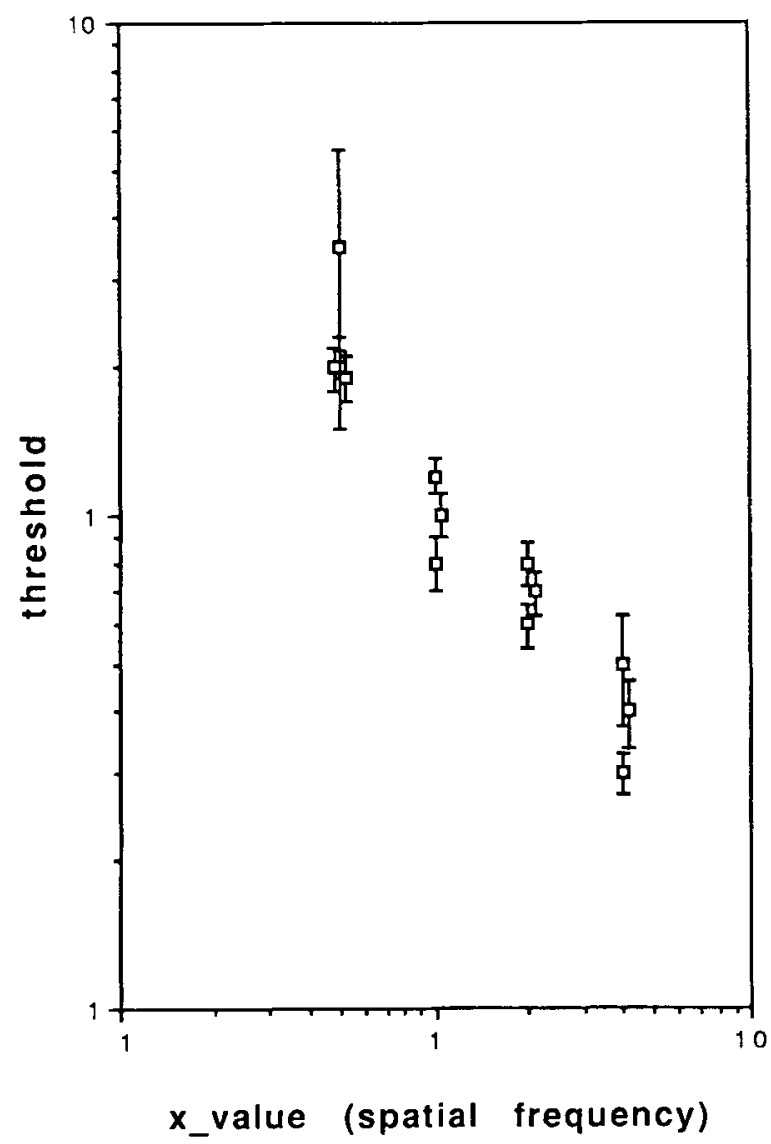

Figure 2. A plot of the data from Figure 1. Several of the data points have been displaced by a small horizontal amount to avoid overlap.
The transformation should be chosen so that the transformed data have equal $S E$ s. The simplest example is shown in Example 3, where the $S E$ of the original data is proportional to the data (multiplicative noise). Because the logarithm of the data is used, the $S E$ s of the transformed data are equal. In Example 4, a power transformation of the data with an exponent of -2 equalizes the $S E s$. The macro could easily be modified to handle other types of transformations.

\section{The Averaging Algorithm}

Step 1: The transformation. The first step is to transform the data so that the main noise contribution is additive and is approximately Gaussian. The macro is able to carry out a power function transformation, whereby

$$
y_{i}=t_{i}^{n}
$$

and

$$
S E_{i}=n t_{i}^{n-1} s e_{i},
$$

where $t_{i}$ is the raw threshold as in Equation 1, and $y_{i}$ is the transformed data for which the dominant noise is assumed to be additive and Gaussian. The choice of $n$ (whether to do arithmetic, geometric, or some other transformation) should be based on experience with the pattern of errors found across many sets of data, and it should not depend on the less reliable pattern of errors found in a single data set.

Equation 8 comes from taking the derivative of Equation 7. From now on, we will use the convention that se in lowercase is the standard error before the transformation and $S E$ in uppercase is the standard error of the transformed variable (Bevington, 1969).

As was discussed in connection with Example 3 above, a logarithmic transformation is often useful. It turns out that the logarithmic transformation is equivalent to a power transformation in the limit as $n$ approaches zero. This can be seen from

$$
\begin{aligned}
t^{n} & =\exp (n \ln t) \\
& =1+n \ln t+(n \ln t)^{2} / 2 !+\ldots \\
& \approx 1+n \ln t
\end{aligned}
$$

as $n$ approaches zero. Thus, averaging $\ln (t)$ is the same as averaging $t^{n}$ in the limit as $n$ goes to zero. In the macro, we replace $n=0$ with $n=.0001$, since $n=0$ would cause $S E$ to vanish in Equation 8. The error in replacing $\ln (t)$ with $t^{n}$ is very small. As an example of using Equation 7 (and Equation 13 for the inverse transformation), suppose we wanted to take the geometric average of 1 and 4. Use of the $\log$ transform gives $y_{\text {ave }}=[\ln (1)+$ $\ln (4)] / 2=0.693147181$. Inversion of this expression gives $t_{\text {ave }}=\exp (0.693147181)=2$. Use of the power transform with $n=.0001$ gives $t_{\text {ave }}=\left(1^{.0001}+4^{.0001}\right) / 2$ $=1.00006932$. The inverse relation (see Equation 13) is $y=[1.00006932]^{10,000}=2.000048046$ (based on the round-off error of Excel). Thus the power transformation with $n=.0001$ is seen to be a close approximation 
to the $\log$ transform. The value of .0001 was chosen as a compromise between having a number close to zero and yet avoiding excessive round-off error. In the section titled Operating the Program, I describe how the macro can be modified to change the transformation so that a true log transform (or any other transformation) can be made.

The transformation exponent, $n$, is entered in the cell above the first $x$ __value (cell A2 of the spreadsheet in Figure 1), with the value of zero representing a logarithmic transformation.

Step 2: Weighted averages. As discussed in connection with Example 1, in order to emphasize the higher quality data, it is preferable to use weighted rather than straight averages. The recommended procedure for a weighted average (Bevington, 1969; Press, Flannery, Teukolsky, \& Vetterling, 1986) is to use the inverse variance as a weighting factor:

$$
1 / \operatorname{Var}_{\mathrm{ave}}=\sum 1 / \operatorname{Var}_{i}
$$

and

$$
y_{\text {ave }}=\sum\left(y_{i} / \operatorname{Var}_{i}\right) \operatorname{Var}_{\text {ave }},
$$

where $\operatorname{Var}_{i}$ is the square of the $S E$ of the $i$ th run. Bevington shows that the value of $y_{\text {ave }}$ given hy Equation 11 minimizes chi-square. The $S E$ of the average is given by

$$
S E_{\text {ave }}=\sqrt{\text { Varave }_{\text {ave }}} .
$$

Step 3: Inverting the transformation. After Equations 10-12 have been applied to get the averages, the transformation of Equations 7 and 8 can be inverted to get back to the average threshold:

$$
t_{\mathrm{ave}}=y_{\mathrm{ave}}{ }^{1 / n}
$$

and

$$
s e_{\mathrm{ave}}=S E_{\mathrm{ave}} n^{-1} t_{\mathrm{ave}}{ }^{1-n} .
$$

Cells B4 and B10 of the macro in Figure 3 calculate Equations 13 and 14. The factor $n^{-1}$ of Equation 14 is not included in the macro, because the factor, $n$, in Equation 8 was not included in cell A16, which produced the original transformation. With $n$ ignored in both places, the calculation is slightly simplified.

Step 4: The heterogeneity factor. The formula for the average variance given by Equation 10 would be inappropriate for the data in Example 2, since it does not include the heterogeneity of the data. The standard correction term, called the heterogeneity factor (Finney, 1971), is to multiply the variance by the reduced chi-square, $\chi_{r}^{2}$ (Bevington, 1969; Press et al., 1986), where $\chi_{r}^{2}$ is given by

$$
\chi_{r}^{2}=\left[\left(\sum_{i}\left(y_{i}-y_{\mathrm{ave}}\right)^{2} / \mathrm{Var}_{i}\right)\right] / d f,
$$

where the degrees of freedom, $d f$, in the denominator are the number of runs minus one. The estimated value, $y_{\text {ave }}$, as given by Equation 11 , is precisely the value that minimizes chi-square.

In the following Excel program, the output consists of four columns for each $x$ _value: $x \_$value, $y_{\text {ave }}, s e_{\text {het }}$, and $\chi_{r}^{2}$, where

$$
s e_{\text {het }}=s e_{\text {ave }} \text { for } \chi_{r}^{2} \leq 1 \text {, }
$$

and

$$
s e_{\text {het }}=s e_{\text {ave }} \sqrt{\chi_{r}^{2}} \text { for } \chi_{r}^{2} \geq 1,
$$

where $s e_{\text {ave }}$ is from Equation 14.

The decision, in Equations 16 and 17, on whether to use the heterogeneity factor (the square root of Equation 15) is not based on any deep theory. We simply choose to be conservative and use whichever definition (Equation 16 or 17) gives the maximum error. The rationale is that we assume that a $\chi_{r}^{2}$ value of less than unity must have occurred by a coincidental agreement between the results of several runs and is not to be trusted. On the other hand, we do trust values of $\chi_{r}^{2}>1$, since there are many good reasons for why the heterogeneity factor can be greater than unity (see Example 2). In our experience, the heterogeneity of the data depends on the experience of the observer. The average reduced chi-square for naive observers is often between 3 and 4 , whereas for trained observers it is often between 1.5 and 2 .

To clarify the heterogeneity factor, consider the average of $1 \pm .1$ and $1.6 \pm .1$. The Excel macro gives an average of $1.3 \pm .3$, with a reduced chi-square of 18 . The se is equal to the product of $s e_{\text {ave }}=.1 \sqrt{2}$ (Equation 12) times the heterogeneity factor of $\sqrt{18}$. Note that this se given by by Equation $17\left(s e_{\text {het }}=.3\right.$ ) is exactly what would have been obtained by the standard root mean square formula if the two numbers 1.0 and 1.6 had been averaged, ignoring the $s e=.1$ of the original data. This simple result is obtained because both of the data had the same ses and because the reduced chi-square was greater than 1 .

It is not always appropriate to use the heterogeneity factor as it is given by Equation 17. Consider the following data, in which the $x_{\text {_n}}$ values are 1,2 , and 3 . Suppose that on Day 1, the $y_{-}$values are $1 \pm .1,2.1 \pm .1$, and $3 \pm .1$,

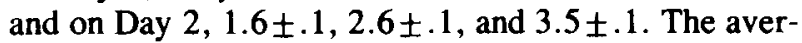
aged data values as calculated by the Excel macro are $1.3 \pm .3,2.35 \pm .25$, and 3.25 , with reduced chi-squares of $18,12.5$, and 12.5. The data for the $x_{\text {_ }}$ value of 1 are the same as those in the preceding paragraph. Suppose that the averaged data are fit by a straight line, using linear regression analysis. Let us now make the critical, but reasonable, assumption that there is additive day-to-day heterogeneity in the data. That is, we assume that $n_{\mathrm{b}}$ in Equation 1 varies between days but not during a single day. In the linear regression fit $y=a x+b$, the $y$ intercept, $b$, should include the heterogeneity factor, but the slope, $a$, should not, because only the intercept and not the slope varies from day to day. One way to proceed is to do the linear regression twice, in which the ses are either $s e_{\text {ave }}$ (for estimating $s e_{\mathrm{a}}$ ) or $s e_{\text {het }}$ (for estimating $s_{\mathrm{eb}}$ ).

An alternate method of fitting the data is not to average the data at each $x$ value before the regression, but rather to include all the raw data in a regression program with several values of $b$, one for each day, to account for the day-to-day variability. This method commonly appears in statistics texts ("repeated measures"), but it is often avoided in linear regressions, since averaged data-the data that actually get plotted-are easier to work with. 


\begin{tabular}{|c|c|c|c|c|}
\hline & $\mathbf{A}$ & B & $\mathrm{C}$ & $\mathrm{D}$ \\
\hline 1 & AVE & & & \\
\hline 2 & $=\operatorname{RESULT}(64)$ & & & \\
\hline 3 & $=$ ARGUMENT("x_value",64) & output results & & \\
\hline 4 & $=$ ARGUMENT("threshold",64) & $=(A 22 / A 21)^{\wedge}(1 / A 8)$ & & \\
\hline 5 & =ARGUMENT("std.error",64) & $=\operatorname{SQRT}(1 / \mathrm{A} 21)^{\bullet} \mathrm{B} 4^{\wedge}(1-\mathrm{A} 7)$ & & \\
\hline 6 & $=$ ROWS $(x$ _value $)$ & $=I F\left(A 20<2,0,\left(A 23-A 22^{\wedge} 2 / A 21\right) /(A 20-1)\right) / A 8^{\wedge} 2$ & & \\
\hline 7 & $=$ INDEX $\left(x_{-}\right.$value, 1$)$ & $=\mathrm{IF}\left(\mathrm{B} 6>1, \mathrm{~B} 5^{*} \mathrm{SQRT}(\mathrm{B} 6), \mathrm{B} 5\right)$ & & \\
\hline 8 & $=I F(A 7=0,0.0001, A 7)$ & & & \\
\hline 9 & $=S E T \cdot V A L U E(B 17.0)$ & $=S E T$ VALUE $($ OFFSET $(A 26, B 17,1), B 4)$ & & \\
\hline 10 & $=F O R\left({ }^{\prime \prime}\right.$ count" $\left.t^{*}, 2, A 6,1\right)$ & $=S E T, V A L U E(O F F S E T(A 26, B 17,2), B 7)$ & & \\
\hline 11 & $=$ INDEX $\left(x_{\text {_value }}\right.$,count $)$ & $=S E T$ VALUE(OFFSET $(A 26,817,3), 86)$ & & \\
\hline 12 & $=$ IF(NOT $($ ISNUMBER(A11)),GOTO(A24)) & & & \\
\hline 13 & $=$ INDEX (std.error, count) & $=I F($ counk $<=A 6, G O T O(B 17))$ & & \\
\hline 14 & $=$ INDEX (threshold, count) & $=$ RETURN $($ INDEX $($ results, 1,1$):$ INDEX(results, $B 17+1,4))$ & & \\
\hline 15 & $=A 13^{*} A 14^{\wedge}(A 7-1)$ & & & \\
\hline 16 & $=A 14^{\wedge} \mathrm{AB}$ & initialization & & \\
\hline 17 & $=I F($ count $=2, G O T O(B 18))$ & $=\mathrm{B} 17+1$ & & \\
\hline 18 & $=\mid F\left(A 11<>\mid N D E X\left(x_{-}\right.\right.$value,count -1$\left.), \mathrm{GOTO}(\mathrm{B} 4)\right)$ & $=$ SET.VALUE(OFFSET $(A 26, B 17,0), A 11)$ & & \\
\hline 19 & $=1 / A 15^{\wedge} 2$ & & & \\
\hline 20 & $=A 20+1$ & $=S E T, V A L U E(A 20,0)$ & & \\
\hline 21 & $=A 21+A 19$ & $=S E T$.VALUE $(A 21,0)$ & & \\
\hline 22 & $=A 22+A 19^{*} A 16$ & $=S E T \cdot \operatorname{VALUE}(\mathrm{A} 22,0)$ & & \\
\hline 23 & $=A 23+A 19^{*} A 16^{\wedge} 2$ & $=S E T$.VALUE $(A 23,0)$ & & \\
\hline 24 & $=$ NEXT() & & & \\
\hline 25 & $=\mathrm{GOTO}(\mathrm{B} 4)$ & $=G O T O(A 19)$ & & \\
\hline 26 & 0.5 & 2 & 0.115 & 0.354 \\
\hline 27 & 1 & 1 & 0.115 & 4 \\
\hline 28 & 2 & 0.681 & 0.057 & 2.054 \\
\hline 29 & 4 & 0.322 & 0.035 & 2.089 \\
\hline
\end{tabular}

Figure 3. The Excel macro for averaging data. The macro itself is in cells A2-B23. Cell A1 contains the name that is used when the macro is called from the spreadsheet containing the data. The results are temporarily stored in cells A24-D27 before they are returned to the spreadsheet.

The point here is that it is useful to understand the role of the heterogeneity factor when one is dealing with averaged data.

\section{Operating the Program}

Explanation of the macro. The macro is listed in Figure 3 . Figure 4 provides an annotated description of each cell. The results are stored in the macro starting in row 26 before they are passed to the spreadsheet. The section of the macro spreadsheet from A26 to D62 can be blank before the macro is executed, or it may have data from a previous averaging calculation. Column A will contain the stimulus strength. Column B will contain the averaged $y$ values. Column $C$ will contain the averaged se according to Equations 16 and 17. Column D will contain the reduced chi-square ( 0 if there is only one run at that $x_{\text {__value). }}$.

Modifying the transformation. Suppose that for some reason a researcher wanted to use a transformation other than a power function. In particular, suppose one wanted a logarithmic transformation as was discussed earlier. The modifications are straightforward:

In cell A16, place the transformed $y_{\text {_nvalue }}$ (= LN[A14]).

In cell A15, place the transformed $S E$, which is the derivative of the transformation times the se (=A13/A14).

In cell B4, place the inverse $y_{\text {_ value transformation }}$ (= EXP[A22/A21]).

In cell $\mathrm{B} 5$, place the inverse se transformation (= SQRT[1/A21] $\cdot$ B4). Note that cells A15 and B5 are the same as the case with a power exponent (cell A7) of 0 , as was discussed following Equation 9.

Running the program. The following steps must be done to carry out the averaging:

Arrange data in the data spreadsheet as shown in Figure 1 .

1. All the runs with the same $x$ _value must be grouped together in consecutive rows (blank rows, or comments cannot be inserted). Figure 1 contains four sets of data, with each set having a different $x_{-}$value. The Excel 


\begin{tabular}{|c|c|c|c|c|}
\hline & $\bar{A}$ & B & $\mathbf{C}$ & D \\
\hline 1 & Name of macro & \multirow[b]{3}{*}{ output results } & & \\
\hline 2 & define RESULTS to be an array & & & \\
\hline 3 & define X_VALUE to be an array & & & \\
\hline 4 & define THRESHOLD to be an array & \multirow{4}{*}{$\begin{array}{l}\text { the weighted average (invert transformation) } \\
\text { the weighted } S E \text { (invert transformation) } \\
\text { the reduced chi-square } \\
\text { maximum of } S E \text { with or without heterogeneity factor }\end{array}$} & & \\
\hline 5 & define STD. ERROR (SE) to be an array & & & \\
\hline 6 & number of rows being passed & & & \\
\hline 7 & exponent of power transformation (n) & & & \\
\hline 8 & replace $n=0$ with $n=.0001$ & \multirow{3}{*}{$\begin{array}{l}\text { output the averaged THRESHOLD } \\
\text { output the averaged SE } \\
\text { outout the chi square for that } X \text { VALUE }\end{array}$} & & \\
\hline 9 & initialize output counter & & & \\
\hline 10 & \multirow{6}{*}{$\begin{array}{l}\text { begin loop. Count goes over all data } \\
\text { the present X_VALUE level } \\
\text { skip if present X_VALUE isn't a number } \\
\text { the present SE of datum } \\
\text { the present datum (THRESHOLD) } \\
\text { SE after transformation } \\
\text { transformed THRESHOLD (y_value) }\end{array}$} & & & \\
\hline$\frac{11}{12}$ & & output the chi square for that $X_{-}$VALUE & & \\
\hline 13 & & if not yet finished initialize for next X_VALUE & & \\
\hline 14 & & exit macro if finished & & \\
\hline 15 & & & & \\
\hline 16 & & initialization & & \\
\hline$\frac{17}{18}$ & $\begin{array}{l}\text { on very first iteration initialize summation } \\
\text { if } X_{\text {_VALUE changes goto output results }} \\
\text { the weighting is inverse variance }\end{array}$ & $\begin{array}{l}\text { increment the number of } X_{\text {_VALUE levels }} \\
\text { output the } X_{\text {_VALUE starting at cell A26 }}\end{array}$ & & \\
\hline 20 & count number of runs at this $x_{\text {_ }}$ value & \multirow{5}{*}{$\begin{array}{l}\text { clear the run counter } \\
\text { clear the weight accumulator } \\
\text { clear the average accumulator } \\
\text { clear the average squared accumulator } \\
\text { start accumulating }\end{array}$} & & \\
\hline 21 & summate weightings & & & \\
\hline 22 & summate weighted y values & & & \\
\hline$\frac{23}{24}$ & $\begin{array}{l}\text { summate weighted y values squared } \\
\text { iterate loop }\end{array}$ & & & \\
\hline 25 & output results when finished & & & \\
\hline 26 & Beginning of $x$ value results & beginning of THRESHOLD results & SE & Chisq \\
\hline
\end{tabular}

Figure 4. A cell-by-cell description of each entry in the macro. The capitalized items, X_VALUE, THRESHOLD, and STD. ERROR are vectors passed to the macro from the spreadsheet. The array RESULTS is returned from the macro to the spreadsheet.

macro will produce four averages. If one wanted to average all 13 data points together, all the $\mathrm{x}$ _values would have to be made equal.

2. The relevant information must occur in three columns that need not be contiguous.

3. There may be gaps between different conditions in the threshold and the $S E$ columns, but in the $x_{\text {_n value }}$ column, the gap cannot be blank; it must be filled with non-numeric text (see Figure 1).

4. The very top number in the $x$ _value column must be the exponent of the transformation in Equation 7.

5. There may be groups of runs with the same

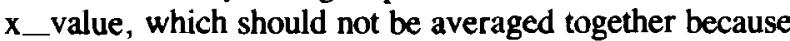
of differences in other parameters. This can be accomplished through the insertion of a gap, or through changing the $x_{\text {_n}}$ value of the second group by an insignificant amount.

Arrange the windows and file names. Two windows must be open: the window with the spreadsheet data should be in front and the macro in back. The following steps are all done on the data spreadsheet. Both programs should reside in the same folder. The macro file should be titled AVERAGE.MACRO.
Select space for results. Find an unused region of the spreadsheet. Select an area four columns wide and at least as many rows as there are different $x_{\text {__ }}$ values to be averaged. Selecting an area means darkening it with the mouse.

Type the macro command. Type in the following in the upper left cell of the selected region, and do not hit the return key:

$$
\text { = AVERAGE.MACRO!AVE(A2:A18,C2:C18,D2:D18) }
$$

The information before the exclamation mark is the file name of the macro. The word after the exclamation mark is the name of the macro, which is located in cell Al of the macro sheet. The items in parentheses are the locations of the $x$ _values, thresholds, and ses. The values can be obtained by dragging the mouse over the relevant columns. The values shown here correspond to the information in Figure 1. Note that the first row is the row containing the exponent (row 2), which is the row above the data.

Execute the macro. Since the calculation returns an array, the macro command must cover all selected cells. On the Macintosh, this is accomplished by not hitting 
return, but rather by depressing the cloverleaf key and simultaneously hitting the enter key. On the IBM version of Excel, the array is selected by simultaneously hitting the control, shift, and enter keys. The calculation will now proceed automatically.

\section{Interpreting the Results}

Columns E-H of Figure 1 present the averages of the data that appear in columns A, C, and D. Three averages were done with transformation exponents of $n=1,0$, and -2 . The exponent $n=1$ (rows 4-7) corresponds to arithmetic averaging. The data with an $\mathrm{x}$ _value of .5 (row 4) produced an average of $2.00 \pm .12$. The value 2.00 makes sense because the first three data-2.1, 1.9, and 2.0 -had equal ses so that their average value was 2.0 , and the outlier $-3.5 \pm 2.0$ - had a much larger se than the others so that it can be ignored. The se of the average (.12) given by Equations 12 and 14 is obtained by dividing the individual ses by $\sqrt{3}$. The heterogeneity factor was not used in calculating the $s e$, because the reduced chi-square of .35 , given in column $H$, is less than unity. For the data with an $x$ _value of 1 (row 5), the average is $1.00 \pm .12$. Equations 12 and 14 would predict that the se should be $.1 / \sqrt{3}=.058$. However, the thresholds of $1.2, .8$, and 1.0 had a larger spread than was expected from the ses, so heterogeneity was present, as is indicated by the reduced chi-square of 4.0. The se of .12 in column $G$ is obtained by multiplying .058 by 2 , which is the square root of the reduced chi-square (the heterogeneity factor).

The results in rows 9-12 are for geometric averaging of the same data. This was achieved by simply changing the exponent in cell A2 from 1 to 0 . The output results change automatically. We were able to present both sets of results in Figure 1 by first converting the previous results to values using the Paste Special feature of Excel. The geometric averages are slightly larger than the arithmetic averages. This is expected on the basis of Equation 8, which causes the weighting to be larger (smaller $S E$ ) for the data with bigger magnitude. For the data in Figure 1, the difference between arithmetic and geometric averaging is quite small because there was only a $40 \%$ difference between the highest and lowest points. With a bigger range, the difference between the two averages can get large. For example, the average of $1 \pm .5$ and $4 \pm 2$ is as follows:

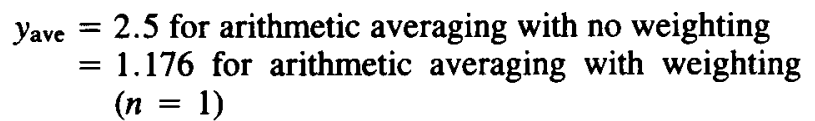

$=2.0$ for geometric averaging with or without weighting $(n=0)$

$=3.4$ for averaging the reciprocal with weighting $(n=-1)$.

Thus, if the numbers span a wide range, the type of averaging can make a big difference. This averaging macro allows the researcher to use the type of averaging that is appropriate to the conditions of the experiment.

The output of the macro above is a set of numbers (the average, se, and reduced chi-square for each condition). A fancier version of this program has been developed that plots the data. The chart or curve information would be numbers like those shown in column B of Figure 1, which identify different conditions (treatments). The macro will produce any number of charts with any number of lines per chart. This program together with documentation also is available on request.

\section{Availability}

There are three ways to obtain the program: (1) Send a blank disk to the author and specify whether it is for an IBM or a Macintosh; (2) type Figure 3 into your computer. (If you choose this method, two names must be defined with the Define Names option in the Formula menu of Excel: AVE must be defined to be in cell A1, and RESULTS must be defined to be in the range A26:D62. D62 can be replaced by $D 100$ or any other row number, de-

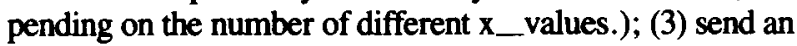
e-mail request to klein@garnet.berkeley.edu. I will e-mail the macro as a text file. The Define Names procedure discussed above must be followed in this case.

\section{REFERENCES}

Bevington, P. R. (1969). Data reduction and error analysis for the physical sciences. New York: McGraw-Hill.

FinNey, D. J. (1971). Probit analysis. Cambridge: Cambridge University Press.

Klein, S. A., \& Stromeyer, C. F. (1980). On inhibition between spatial frequency channels: Adaptation to complex gratings. Vision Research, 20, 459-466.

Klein, S. A., Stromeyer, C. F., \& Ganz, L. (1974). The simultaneous spatial frequency shift: A dissociation between the detection and perception of gratings. Vision Research, 14, 1421-1432.

Press, W. H., Flannery, B. P., Teukolsky, S. A., \& Vetterling, W. T. (1986). Numerical recipes. Cambridge: Cambridge University Press.

(Manuscript received December 31, 1990; revision accepted for publication July 23, 1991.) 